\title{
Convergent Calculations That Dark Solutions Are Reflective of Mass-Energy yet to Occur
}

\author{
Michael A. Persinger \\ Laurentian University, Sudbury, Canada \\ Email: mpersinger@laurentian.ca
}

Received February 4, 2012; revised March 15, 2012; accepted March 22, 2012

\begin{abstract}
The discrepancy between the observed and expected estimates from universal constants of mass-energy within the universe is in the order of a factor of $\sim 10$. Discrepancies between numerical solutions between the models of Dirac, Szydowski-Godlowski, and Friedman could be accommodated by the gnomonic solution of 0.44 for a square that displays both linearity and curvature (flare). This value is also reflected in dimensionless parameter A, the term for 4D-G (gravitational constant) transformation, and the optimal $\mathrm{k}$ in Friedman's universe. One interpretation from Go (density), assuming an effective average mass of 1 proton $/ \mathrm{m}^{3}$ as a universal, one-particle force, is that dark solutions reflect the matter yet to occur in the open cold matter model of $\sim 90$ billion years.
\end{abstract}

Keywords: Dark Matter; Gravity; Physical Cosmology; Square Gnomon; Age of Universe

\section{Introduction}

The accommodation of dark matter and energy within contemporary values for the constraints from constants and known space-time boundaries has been subjected to multiple formulations [1,2]. Two central contradictions are the discrepancy by a factor of $\sim 10$ between age of the universe and its estimated mass and density and the simultaneous requirement for both curvature and noncurvature for its shape. In the present paper a potential explanation for the "origin" of dark matter and how it might relate to a mathematical form that intrinsically contains properties of both curvature and linearity is explored.

\section{The Dimensionless Constant in Dynamic Pressure to G Conversion}

Paul Dirac [3] suggested that within a zero-curvature universe the mass would be finite and could be described as a large dimensionless number of $10^{78}$. Assuming a unit proton per $\mathrm{m}^{3}$, which is consistent with a pressure of an effective one-particle force [1], Persinger [4] estimated the intrinsic universal pressure $\left(\partial \mathrm{c}^{2}\right)$, where $\partial$ is density and $\mathrm{c}=$ velocity of light) of $1.5 \times 10^{-10} \mathrm{~kg} / \mathrm{m} \cdot \mathrm{s}^{2}$. The transform required for an equivalence to $G$, the gravitational constant $\left(\mathrm{m}^{3} \mathrm{~kg} \cdot \mathrm{s}\right)$, was $\mathrm{m}^{4} / \mathrm{kg}^{2}$. Assuming the width of the universe to be $\sim 10^{26} \mathrm{~m}(1 / \sqrt{\Lambda}$, the cosmological constant), the value for $\mathrm{m}^{4}$ is $10^{104}$ (an extra dimension to $10^{78}$ ) and hence the mass would be $10^{52} \mathrm{~kg}$. If Dirac's number represented three-dimensional space and there was an average of 1 proton $/ \mathrm{m}^{3}$ the mass would be about $10 \%\left(10^{51} \mathrm{~kg}\right)$ of that estimate.

However to obtain the equivalence of coefficients between the estimated intrinsic "dynamic pressure" and G, the transformation term was $0.44 \mathrm{~m}^{4} / \mathrm{kg}^{2}$ which is remarkably similar to the dimensionless parameter A [4] which has been calculated to average 0.46 with a lower range that would include 0.44 . The parameter has been employed to constrain models for dark energy and reflects a peak observed for baryonic acoustic oscillations [2]. One the consequences of this term when included in various models of the density parameter $\Omega$, the ratio of the observed density $(\partial)$ to the critical density $\partial_{c}$ of a Friedmann-type universe, is that the universe is almost flat. The typical equation for the density parameter is:

$$
\Omega=(8 \pi G \partial) / 3 \mathrm{H}^{2}
$$

where $\mathrm{H}$ is Hubble's parameter.

In numbers the quantity $8 \times 3.14 \times 6.67 \times 10^{-11} \mathrm{~m}^{3} / \mathrm{kg}$ $\mathrm{s}^{2} \times 0.2 \times 1.67 \times 10^{-27} \mathrm{~kg} / \mathrm{m}^{3}$ divided by $3 \times 5.89 \times 10^{-36}$ $\mathrm{s}^{-2}$ (assuming $75 \mathrm{~km} / \mathrm{s} / \mathrm{MParsec}$ ), i.e. $\mathrm{h}=2.4 \times 10^{-18} \mathrm{~s}^{-1}$, is 0.032 . This is a factor of 10 smaller than the precise model of Syzdlowski and Godlowski [5] whose $\Omega_{\mathrm{m}, 0}$ solution was a concentric centroid of 0.30 . Due to primordial nucleosynthesis they assumed that for $\mathrm{h}=1\left(\mathrm{H}_{0}=\right.$ $100 \mathrm{~km} / \mathrm{s} / \mathrm{MParsec}$ ) baryonic mater would constitute a term of 0.05 indicating that the greatest proportion of their $\Omega_{\mathrm{m}, 0}$ solution is from non-baryonic dark matter. This approximately 10 fold difference is consistent with the discrepancy between the mass-energy equivalent pre- 
dicted by Dirac's number and the value derived from density matched to $\mathrm{G}$.

\section{Friedman's Expansion and $\sim 0.44$}

Friedman's conceptualization of the expansion of the universe, Hubble's constant, was

$$
\mathrm{H}^{2}=[(G 8 \pi / 3) \cdot \partial]\left[-\left(\mathrm{k} / \mathrm{a}^{2}\right)\right]
$$

(the latter term being the product of the inverse of Lambda-the cosmological constant) and $\mathrm{k}$, intrinsic curvature). The square root of $\mathrm{H}$ is $9 \times 10^{7} \mathrm{~m} / \mathrm{s}$. However Hubble's constant is per MegaParsec and there are $3.1 \times$ $10^{22} \mathrm{~m} / \mathrm{MPsec}$. From an estimated universal width of $10^{26}$ $\mathrm{m}$, the universe is $\sim 3000$ MPsec. $\sqrt{\mathrm{H}}\left(9 \times 10^{7} \mathrm{~m} / \mathrm{s}\right) \mathrm{di}-$ vided by $3 \times 10^{3}$ MPsec, is $3 \times 10^{4} \mathrm{~m} / \mathrm{s}$ or $30 \mathrm{~km} / \mathrm{s}$. The current estimated mean value for Hubble's constant, empirically, is $75 \mathrm{~km} / \mathrm{s}$. The curvature value is $30 \mathrm{~km} / \mathrm{s}$ divided by 75 or 0.40 . This value is within measurement variability of 0.44 . If 0.44 were employed as the reference the velocity in this model would be $\sim 68 \mathrm{~km} / \mathrm{s} /$ MParsec.

\section{The Congruent Gnomon Solution}

There have been many thoughtful explorations of complex geometries to accommodate the universe's shape [e.g., 6-11]. A less mathematically complex potential solution is the gnomon, which is a form that when added to some form results in a new form similar but not identical to the original [12]. The flare (or curvature of spiral) solution to a square winkle's gnomon is $\lambda=$ $2 / \pi \cdot \ln 2=0.44$, a logarithmic spiral that circumscribes the self-similarity of an infinite constructive process. Although infinite, the process is bounded with finite perimeters. This value is also within the range of the dimensionless constant.

The consideration of a "flat" square and its intrinsic curvature might also be a candidate to explain the properties of basic forces. The essence of squares is their symmetrical right angles which dominate the conceptualization of both Euclidean and non-Euclidean space. Superimposed upon this primary would be the non-linear component of an expanding curvature perceived as a spiral whose radius doubles every $90^{\circ}$ that would be required for the exactness of any calculations. Depending upon the assumptions of the organization of structure between Planck's length and that occupied by matter $\left(\sim 10^{-15} \mathrm{~m}\right)$ the integrated finite perimeter would be between about a factor of 16 and $8 \pi$ longer with 6 additional levels but wrapped within four-dimensional space.

\section{Dark Matter as Potential Matter}

Solutions that assume limits or boundaries of a system can be more amenable to its parsimonious representation because the dynamics become static. Across levels of scientific discourse [13] there is a clear relationship between the absolute values of the $\Delta \mathrm{s}$ (the increment of space) being measured to discern a phenomenon and the optimal $\Delta t$ (increment of time) required for it to be observed (measured) as an integral unit. As defined by the Nyquist limit the threshold for discerning a process must be $>2 \Delta t$. At the maximum boundary, the universe, where both $\Delta \mathrm{s}$ and $\Delta \mathrm{t}=1$, this would not occur. In other words there would be no process operations that usually complicate the geometry and the temporal properties or relationships between units of matter. The universe would be static (fixed) because there would be no time.

The most parsimonious relationship that defines when this maximum time might occur is $\partial \mathrm{G}$. Assuming a density of one proton per unit $\mathrm{m}^{3}$ the quantity is $(1.61 \times$ $\left.10^{-27} \mathrm{~kg} / \mathrm{m}^{3}\right) \times 6.67 \times 10^{-11} \mathrm{~m}^{3} / \mathrm{kg} \cdot \mathrm{s}^{2}$ or $10.74 \times 10^{-38} \mathrm{~Hz}^{2}$ or the equivalent of $3 \times 10^{18} \mathrm{~s}$ ( 90 billion years). This value is similar to estimates by Hoffman et al. [6] who calculated that in the open cold matter (OCDM) model the boundary for the fate of the universe (the final epoch) would be 89.2 billon years. Assuming the contemporary outer range of the age of the universe to be 13 billions of years or $4.1 \times 10^{17} \mathrm{~s}$, this would indicate that the current formation is $\sim 14 \%$ of the ultimate boundary condition. Ordinary baryonic matter accounts for $10 \%$ to $20 \%$ [14] of the masses of major galactic clusters which have been attributed to "dark matter".

One interpretation of this congruence is that the energy-mass equivalence attributed to smaller increments of matter might be applied to the entire space occupied by all mass. If there is potential energy then the presence of potential mass, not yet manifested, is one implication. From this perspective the influences attributed to "dark matter" reflect the matter yet to be formed within an expanding universe. The involvement of the total time of the universe within which there is no process would also be consistent with the suggestion by Balakin et al. [1] that tachyon matter is a candidate for dark matter and energy.

\section{The Casimir Contribution}

One of the assumptions of the Casimir effect [15] is matter is formed from virtual particles within the vacuum potential if the boundary of an electromagnetic field is expanding. The challenge to an expanding universal boundary and the matter within it is often facilitated by demonstrating a coupling between gravitational and electrodynamic processes. Assuming the intrinsic pressure within the universe to be $15 \times 10^{-11} \mathrm{~Pa}$ [4] and to equate $\partial \mathrm{c}^{2}$ with $\mathrm{G}$ and the existence of a concentric second boundary around the universal boundary that acts against passive expansion, the separation between the two neu- 
tral boundaries would be:

$$
\mathrm{a}=\left[-\left(\pi^{2} \hbar \mathrm{c}\right) \mathrm{S} /(240 \mathrm{~F})\right]^{1 / 4}
$$

where $\hbar$ is the modified Planck's constant, F is the force derived from $\partial \mathrm{c}^{2}$ and $\mathrm{S}$ is the surface area of the universe assuming a circumference is between $10^{26}$ to $10^{27} \mathrm{~m}$. The resulting separation between these two concentric surfaces of the universal boundary would be $54 \mu \mathrm{m}$ [16]. Assuming this thin shell is a black body the equivalent temperature from Wein's law is $53^{\circ} \mathrm{K}$ and according to Stephan's law, the power density would be the product of $\mathrm{T}^{4}$ and the constant $5.67 \times 10^{-8} \mathrm{~W} / \mathrm{m}^{2}$, or $0.45 \mathrm{~W} / \mathrm{m}^{2}$.

If Varshni's [17] assessment of the distribution of redand blue-shift data are applicable, that is our solar system is near the center of distributions of galaxies and by inference the universe, then one could assume that the energy generated by and throughout the black body shell produced by the Casimir effect would decrease as a function of $\mathrm{r}^{-1}$ rather than $\mathrm{r}^{-2}$ in all directions. Hence, the power density reaching radio telescopes would be $(0.45$ $\left.\mathrm{W} / \mathrm{m}^{2}\right) / \sim 10^{26} \mathrm{~m}$ or in the order of hundreds of mJy $(1$ Janksy $\left.=10^{-26} \mathrm{~W} / \mathrm{m}^{2} \mathrm{~Hz}\right)$. If the Casimir pressure $(1.5 \times$ $10^{-10} \mathrm{~Pa}$ or $\left.\mathrm{kg} / \mathrm{m} \cdot \mathrm{s}^{2}\right)$ is multiplied by the estimated volume of the universe $\left(\sim 10^{78} \mathrm{~m}^{3}\right)$ the energy is $1.5 \times 10^{68} \mathrm{~J}$ or about $10 \%$ of the total energy-mass equivalence based upon $10^{52} \mathrm{~kg}$ or $8 \pi \mathrm{G} / \mathrm{c}^{4} \cdot \mathrm{T}_{\mathrm{uv}}$.

The implications for these solutions is that with the presence of $0.45 \mathrm{~W} / \mathrm{m}^{2}$ and an estimated universal surface area of $4.5 \times 10^{53} \mathrm{~m}^{2}$ there would $\sim 2 \times 10^{53} \mathrm{~J} / \mathrm{s}$ available with a mass equivalence of $2 \times 10^{36} \mathrm{~kg}$ or about 1 million solar masses per sec. At this rate the current mass would be matched within $10^{16} \mathrm{~s}$ or within a factor of 0.1 of the current age of the universe. This rate of acceleration is well within the range expected within the SzydlowskiGodlowski model [5]. The transformation of virtual particles to "real" particles by the Casimir process not only reiterates the intricate connection between it and G [18] but suggests that dark matter and energy would be our present inferential measurement of the virtual condition.

\section{Conclusion}

The accommodation of the approximately $10 \%$ discrepancy between expected and observed mass-energy equivalents has been considered the basis for the presumption of dark matter and energy which has been interconnected with spatial curvature and one particle forces. The simultaneous accommodation of linear and non-linear geometry might be accomplished by the systematic application of the gnomonic solution for the square of 0.44 which is a value that solves for several proportions including the Friedmann curvature $\mathrm{k}$ and dimensionless parameter A. One possible interpretation of the results developed in this paper is that dark matter and energy are manifestations from Casimir virtual particles of what is yet to occur within a system determined by $\mathrm{G}$ within a OCDM model of the universe whose fate is $\sim 90$ billion years.

\section{Acknowledgements}

Thanks to Blake T. Dotta, Lucas Tessaro and Ghislaine F. Lafreniere for technical comments concerning the manuscript.

\section{REFERENCES}

[1] A. B. Balakin, D. Pavon, D. J. Schwarz and W. Zimdahl, "Curvature Force and Dark Energy," New Journal of Physics, Vol. 5, 2003, pp. 85.1-85.14.

[2] P. Wu and H. Yu, "Reconstructing the Properties of Dark Energy from Recent Observations," Journal of Cosmology and Astroparticle Physics, Vol. 10, 2007, pp. 1-13.

[3] P. Dirac, "The Principles of Quantum Mechanics," Oxford Press, Oxford, 1947.

[4] M. A. Persinger, "A Simple Estimate of the Mass of the Universe: Dimensionaless Parameter A and the Construct of 'Pressure'," Journal of Physics, Astrophysics and Physical Cosmology, Vol. 3, No. 1, 2009, pp. 1-3.

[5] M. Szydlowski and W. Godlowski, "Acceleration of the Universe Driven by Casimir Force," International Journal of Modern Physics D, Vol. 17, No. 2, 2008, pp. 343366. doi:10.1142/S021827180801205X

[6] Y. Hoffman, O. Lahav, G. Yepes and Y. Dover, "The Future of the Local Large Scale Universe: The Roles of Dark Matter and Dark Energy," Journal of Cosmology and Astroparticle Physics, Vol. 10, 2007, pp. 1-16.

[7] V. Petkov, "On the Reality of Minkowski Space," Foundations of Physics, Vol. 37, No. 10, 2007, pp. 1499-1502. doi:10.1007/s10701-007-9178-9

[8] H. Cheng, "The Asymptotic Behavior of Casimir Force in the Presence of Compactified Universal Extra Dimensions," Physics Letters B, Vol. 643, No. 6, 2006, pp. 311314. doi:10.1016/j.physletb.2006.10.051

[9] M. Y. Konstantinov, "Topological Transitions and LargeScale Structure of Space-Time in Multidimensional Theory of Gravity," Russian Physics Journal, Vol. 40, No. 2, 1997, pp. 124-128. doi:10.1007/BF02806177

[10] J. Audretsch, "Riemannian Structure of Space-Time as a Consequence of Quantum Mechanics," Physics Review D, Vol. 27, No. 12, 1983, pp. 2872-2883. doi:10.1103/PhysRevD.27.2872

[11] L. P. Eisenhart, "A Unified Theory of General Relativity of Gravitation and Electromagnetism. IV," Proceedings of the National Academy of Sciences, Vol. 43, No. 4, 1957, pp. 333-336. doi:10.1073/pnas.43.4.333

[12] M. J. Gazele, “Gnomon," Princeton U. Press, Princeton, 1999.

[13] M. A. Persinger, "On the Nature of Space-Time Perception of Phenomena in Science," Perceptual and Motor Skills, Vol. 89, 1999, pp. 1210-1216. 
[14] T. Ponman, "The Matter with Density," Science, Vol. 414, 2001, pp. 402-404.

[15] M. Bordag, U. Mohideen and V. M. Mostepanenko, "New Developments in the Casimir Effect," Physics Reports, Vol. 353, No. 1-3, 2001, pp. 1-205. doi:10.1016/S0370-1573(01)00015-1

[16] S. A. Koren and M. A. Persinger, "The Casimir Force along the Universal Boundary: Quantitative Solutions and Implications," Journal of Physics, Astrophysics and Phy- sical Cosmology, Vol. 4. No. 1, 2010, pp. 1-5.

[17] Y. P. Varshni, "The Red Shift Hypothesis for Quasars: Is the Earth the Center of the Universe?" Astrophysics and Space Science, Vol. 43, No. 1, 1976, pp. 3-8. doi:10.1007/BF00640549

[18] H. E. Puthoff, "Gravity as Zero-Point-Fluctuation Force," Physical Review A, Vol. 39, No. 5, 1989, pp. 2333-2342. doi:10.1103/PhysRevA.39.2333 